\section{Características da violência física e sexual contra crianças e adolescentes atendidos no IML de Maceió, Alagoas, Brasil}

\author{
Characteristics of physical and sexual violence \\ against children and adolescents examined at the \\ Forensic Medicine Institute in Maceió, \\ Alagoas State, Brazil
}

1 Universidade Estadual de Ciências da Saúde de Alagoas, Maceió, Brasil. 2 Instituto Médico Legal de Maceió, Maceió, Brasil. 3 Universidade Federal de São Paulo, São Paulo, Brasil.

Correspondência J. A. T. L. Guimarães Universidade Estadual de Ciências da Saúde de Alagoas. Rua Basileu de Meira Barbosa 395, Maceió, AL 57057-480, Brasil.

joaolegista@globo.com

\section{Abstract}

The purpose of this study was to characterize cases of violence against children and adolescents examined at the Forensic Medicine Institute in Maceió, Alagoas State, Brazil. Three hundred and three cases from September, 2008 to March, 2009, were analyzed. Victims were interviewed and data were collected on the type of violence, victim's gender, age, schooling, and economic class, maternal schooling, characteristics of the aggressor and person reporting the case, location, and repeat offenses. Violence against children and adolescents in this sample was concentrated in lower income groups, and girls were victimized more frequently than boys. Most perpetrators were non-relatives, but known to the family. Sexual abuse was more common among younger children, while physical assault was more common against adolescents. The victim's home was the most frequent site of the violence. Further studies are needed to determine whether violence in other socioeconomic groups and against males actually does not occur, or whether it simply is not reported to the Forensic Medicine Institute and thus remains invisible.

Agression; Violence; Sexual Violence; Adolescent; Child
João Alfredo Tenório Lins Guimarães 1,2 Wilza Vieira Villela 3

\section{Introdução}

A repercussão da violência sobre a saúde das coletividades transforma-a em um problema de saúde pública que, como tal, deve ser enfrentado através de esforços coletivos provenientes de setores diversos como educação, saúde, serviço social e justiça. Na perspectiva da concepção ampliada de saúde, a violência torna-se objeto da intersetorialidade, em que o campo médicosocial se integra 1 .

A violência contra crianças e adolescentes se caracteriza como uma situação em que se identifica a existência de um sujeito em condições de superioridade, que cometa dano, seja este corporal, psicológico ou sexual, contrariamente à vontade da vítima ou mesmo após consentimento desta, quando tal experiência for obtida por indução ou sedução enganosa 2 .

Vários autores 2,3 destacam o papel dos abusos físico e sexual no contexto geral das violências praticadas contra o segmento infanto-juvenil, principalmente quando a vítima é do sexo feminino ${ }^{4}$. Violência psicológica, negligência e mitigação de direitos são também encontradas, porém, sua detecção demanda esforços multiprofissionais.

O presente estudo abordou a temática da violência contra a criança e o adolescente, mais especificamente os abusos físicos e sexuais, cuja constatação é realizada no âmbito médico-legal. O objetivo do trabalho foi descrever as caracte- 
rísticas deste tipo de violência praticada contra crianças e adolescentes entre a população atendida no Instituto Médico Legal de Maceió (IML/ Maceió), Alagoas, Brasil.

\section{Métodos}

Após aprovação pelo comitê de ética da Universidade Estadual de Ciências da Saúde de Alagoas (protocolo 699/07), foram realizadas 303 entrevistas com crianças e adolescentes vítimas de violência, encaminhados ao IML/Maceió para exame de corpo de delito no período de agosto/2008 a fevereiro/2009.

As entrevistas foram realizadas pelo pesquisador principal, perito odonto-legal lotado no IML/ Maceió, nas dependências do próprio Instituto, após o exame pericial, na presença dos responsáveis e em sala privativa no setor de Psicologia. Algumas variáveis foram coletadas diretamente dos encaminhamentos dos participantes (autoridade solicitante do exame, sexo, idade, procedência, classificação econômica, tipo, subtipo e local da prática da violência). As demais (cor, ma- trícula escolar e escolaridade da vítima; escolaridade da mãe da vítima; acompanhante durante o exame; denunciante da violência; existência de denúncias adicionais; identificação do agressor) foram coletadas durante as entrevistas.

Considerando critérios do Estatuto da Criança e do Adolescente, os participantes foram alocados em dois grupos: crianças (composto por crianças até 12 anos incompletos) e adolescentes (composto por adolescentes até 18 anos incompletos).

\section{Resultados e discussão}

Neste estudo, o sexo feminino foi preponderante entre crianças e adolescentes vítimas de violência (Tabela 1). Esses achados são consonantes com a literatura e destacam o sexo feminino entre casos de violência contra a criança e o adolescente 5 , principalmente quando levantam características da violência sexual 6 .

A elevada proporção de vítimas oriundas de camadas econômicas mais baixas é uma característica central dessa pesquisa (Tabela 1). Estudos

\section{Tabela 1}

Distribuição de crianças e adolescentes vítimas de violência segundo sexo, cor e classificação econômica. Maceió, Alagoas, Brasil.

\begin{tabular}{|c|c|c|c|c|c|c|}
\hline \multirow[t]{3}{*}{ Variáveis } & \multicolumn{4}{|c|}{ Grupo } & \multicolumn{2}{|c|}{ Total } \\
\hline & \multicolumn{2}{|c|}{ Crianças } & \multicolumn{2}{|c|}{ Adolescentes } & \multirow[b]{2}{*}{$\mathbf{n}$} & \multirow[b]{2}{*}{$\%$} \\
\hline & n & $\%$ & n & $\%$ & & \\
\hline \multicolumn{7}{|l|}{ Sexo } \\
\hline Masculino & 39 & 12,8 & 43 & 14,2 & 82 & 27,0 \\
\hline Feminino & 74 & 24,4 & 147 & 48,6 & 221 & 73,0 \\
\hline Total & 113 & 37,2 & 190 & 62,7 & 303 & 100,0 \\
\hline \multicolumn{7}{|l|}{ Cor } \\
\hline Negro & 9 & 3,0 & 24 & 7,9 & 33 & 10,9 \\
\hline Branco & 28 & 9,2 & 25 & 8,3 & 53 & 17,5 \\
\hline Pardo & 76 & 25,1 & 141 & 46,5 & 217 & 71,6 \\
\hline Total & 113 & 37,3 & 190 & 62,7 & 303 & 100,0 \\
\hline \multicolumn{7}{|c|}{ Classificação econômica } \\
\hline A1 & 1 & 0,3 & 0 & 0,0 & 1 & 0,3 \\
\hline $\mathrm{A} 2$ & 1 & 0,3 & 3 & 1,0 & 4 & 1,3 \\
\hline B1 & 5 & 1,6 & 2 & 0,6 & 7 & 2,2 \\
\hline B2 & 4 & 1,3 & 16 & 5,3 & 20 & 6,6 \\
\hline $\mathrm{C} 1$ & 6 & 2,0 & 21 & 6,9 & 27 & 8,9 \\
\hline $\mathrm{C} 2$ & 22 & 7,3 & 48 & 15,9 & 70 & 23,2 \\
\hline $\mathrm{D}$ & 49 & 16,2 & 72 & 23,8 & 121 & 40,0 \\
\hline$E$ & 25 & 8,3 & 28 & 9,2 & 53 & 17,5 \\
\hline Total & 113 & 37,2 & 190 & 62,7 & 303 & 100,0 \\
\hline
\end{tabular}


têm demonstrado que a violência possui uma lógica social perversa ${ }^{4,7}$. A pequena participação de sujeitos de classes econômicas mais elevadas gera uma reflexão sobre a detecção da violência dentro das classes economicamente mais privilegiadas.

Entre as vítimas encaminhadas ao IML/ Maceió, os achados deste estudo demonstram a predominância da violência entre crianças e adolescentes pardos (Tabela 1). Estudos têm demonstrado que a violência e vitimização seguem um padrão étnico que teriam pardos e negros como destaques 8 . Estudos internacionais tam- bém demonstram algum tipo de associação da vitimização infanto-juvenil em relação à cor, com predominância da raça negra ${ }^{9}$. Em uma capital do nordeste do Brasil, a violência contra crianças e adolescentes foi predominante entre pardos e brancos 10 .

As delegacias de polícia continuam a ser as principais instituições a encaminhar vítimas de violência para exame de corpo de delito (Tabela 2). Embora legalmente habilitado para a solicitação de exames, o Conselho Tutelar acaba desempenhando um papel secundário, atuando mais ativamente nos casos em que as vítimas são

Tabela 2

Distribuição dos participantes por grupo segundo autoridade solicitante do exame, acompanhante, denunciante, agressões e denúncias adicionais de violência. Maceió, Alagoas, Brasil.

\begin{tabular}{|c|c|c|c|c|c|c|}
\hline \multirow[t]{3}{*}{ Variáveis } & \multicolumn{4}{|c|}{ Grupo } & \multicolumn{2}{|c|}{ Total } \\
\hline & \multicolumn{2}{|c|}{ Crianças } & \multicolumn{2}{|c|}{ Adolescentes } & \multirow[b]{2}{*}{$\mathbf{n}$} & \multirow[b]{2}{*}{$\%$} \\
\hline & $\mathbf{n}$ & $\%$ & $\mathbf{n}$ & $\%$ & & \\
\hline \multicolumn{7}{|l|}{ Autoridade solicitante } \\
\hline Conselho Tutelar & 35 & 11,5 & 27 & 8,9 & 62 & 20,4 \\
\hline Delegacia especializada & 45 & 14,9 & 72 & 23,8 & 117 & 34,7 \\
\hline Outras delegacias & 33 & 10,9 & 90 & 29,7 & 123 & 44,6 \\
\hline Ministério Público & 0 & 0,0 & 1 & 0,3 & 1 & 0,3 \\
\hline Total & 113 & 37,3 & 190 & 62,7 & 303 & 100,0 \\
\hline \multicolumn{7}{|l|}{ Acompanhante no exame } \\
\hline Pai/Mãe & 61 & 20,1 & 123 & 40,6 & 184 & 60,7 \\
\hline Familiar diverso & 6 & 2,0 & 26 & 8,6 & 32 & 10,6 \\
\hline Conselheiro & 16 & 5,3 & 20 & 6,6 & 36 & 11,9 \\
\hline Conselheiro + Familiar & 29 & 9,6 & 13 & 4,3 & 42 & 13,9 \\
\hline Outros & 1 & 0,3 & 8 & 2,6 & 9 & 2,9 \\
\hline Total & 113 & 37,3 & 190 & 62,7 & 303 & 100,0 \\
\hline \multicolumn{7}{|l|}{ Denunciante } \\
\hline Pai/Mãe & 69 & 22,8 & 109 & 36,0 & 178 & 58,8 \\
\hline Familiar diverso & 15 & 4,9 & 24 & 7,9 & 39 & 12,8 \\
\hline Conhecido & 4 & 1,3 & 13 & 4,3 & 17 & 5,6 \\
\hline Anônimo & 16 & 5,3 & 13 & 4,3 & 29 & 9,6 \\
\hline Vítima & 0 & 0,0 & 20 & 6,6 & 20 & 6,6 \\
\hline Profissionais de saúde & 4 & 1,3 & 0 & 0,0 & 4 & 1,3 \\
\hline Educadores & 2 & 0,6 & 3 & 1,0 & 5 & 1,6 \\
\hline Outros & 3 & 1,0 & 8 & 2,6 & 11 & 3,6 \\
\hline Total & 113 & 37,3 & 190 & 62,7 & 303 & 100,0 \\
\hline \multicolumn{7}{|l|}{ Agressões adicionais } \\
\hline Sim & 31 & 10,2 & 43 & 14,2 & 74 & 24,4 \\
\hline Não & 82 & 27,1 & 147 & 48,5 & 229 & 75,6 \\
\hline Total & 113 & 37,3 & 190 & 62,7 & 303 & 100,0 \\
\hline \multicolumn{7}{|l|}{ Denúncias adicionais } \\
\hline Sim & 10 & 3,3 & 11 & 3,6 & 21 & 6,9 \\
\hline Não & 103 & 34,0 & 179 & 59,1 & 282 & 93,1 \\
\hline Total & 113 & 37,3 & 190 & 62,7 & 303 & 100,0 \\
\hline
\end{tabular}


crianças. Esse achado é coerente com a proporção de vítimas que são acompanhadas por um conselheiro tutelar durante o exame no IML/Maceió (Tabela 2).

São inúmeras as barreiras para a notificação da violência contra crianças e adolescentes por profissionais de saúde 11 . Neste estudo, a família respondeu pela grande maioria das notificações, ficando profissionais de saúde e de educação em plano secundário, embora haja obrigação legal para estes profissionais.

Concernente à vitimização por agressões adicionais, mais de um quarto das crianças e pouco mais de $22 \%$ dos adolescentes incluídos no estudo relataram ter sido vítimas de outras agressões. Paralelamente a esse resultado, menos de um terço das vítimas de agressões prévias figuraram anteriormente em algum tipo de denúncia às autoridades competentes (Tabela 2).

No tocante à gravidade da violência física, a maioria das lesões foi leve (Tabela 3). Estes achados estão de acordo com a literatura 12 . Principalmente em relação aos abusos sexuais, nem sempre é possível encontrar sinais de violência através do exame médico legal 13. Isso não significa que a criança ou o adolescente não tenha sido vítima de abuso. No presente estudo, entre as vítimas de violência sexual, foi grande a proporção de vítimas que não apresentavam sinais de violência, detectáveis através do exame médico legal (Tabela 3). Nesse sentido, Shrier et al. ${ }^{14}$ alertam para a necessidade de inclusão, na história médica, de relatos sobre atos sexuais forçados ou sob pressão, durante o exame clínico de adolescentes com vida sexual ativa.

Em relação à identificação dos agressores, individualmente, conhecidos fora da família e padrastos são os maiores agressores entre as crianças. Se somadas as agressões praticadas pelo núcleo familiar (pai, mãe, familiares, padrasto), a proporção é muito maior. Entre adolescentes, predominaram agressores conhecidos fora da família, perfil semelhante ao encontrado por Monteiro et al. 15 em Teresina, Piauí. Ainda entre adolescentes, o companheiro apresentou destaque, demonstrando que, com o avanço da idade a violência contra criança ganha contornos de violência doméstica e de gênero.

A literatura refere a casa da vítima como local preferencial para a prática da violência 6,16. No presente estudo, entre as crianças, a casa da vítima foi o local preponderante tanto entre os casos de violência física quanto sexual, revelando que os agressores são pessoas de dentro da família ou têm livre acesso ao interior da casa. Entre os adolescentes, a via pública foi o principal local da prática de agressão física. Isto demonstra que, à medida que ficam mais velhas, as crianças estão expostas a outros tipos de violência, que incluem a violência interpessoal fora da família e a violência da delinquência. Os crimes sexuais entre os adolescentes foram praticados preferencialmente na casa do agressor e casa da vítima, reforçando a ideia de que este tipo de violência ocorre de forma semelhante ao grupo de crianças, ou seja, praticado por pessoas que gozam de certa confiança por parte das vítimas (Tabela 3).

A violência contra o segmento infanto-juvenil é um fenômeno que tem acompanhado a história da humanidade e parece estar ainda enraizado nas sociedades contemporâneas. $\mathrm{O}$ patriarcalismo, a violência de gênero, problemas econômicos, etnia e baixa escolaridade são alguns dos fatores que mantêm estreita relação com a prática da violência contra crianças e adolescentes.

Em um Estado pobre como Alagoas, onde são múltiplas as áreas necessitadas, a deficiência de políticas públicas específicas para a prevenção e erradicação da violência contra crianças e adolescentes é mais um problema complexo que tem demandado enfrentamento prioritário.

Esperamos que este estudo possa contribuir para a descrição, compreensão, reflexão e discussão do fenômeno da violência contra o segmento infanto-juvenil em nosso estado, servindo de apoio para outros autores e para instituições ligadas diretamente à prevenção e erradicação desse problema social complexo. 
Tabela 3

Distribuição dos participantes por grupo segundo tipo, subtipo, gravidade, local da prática da violência e agressor.

Maceió, Alagoas, Brasil.

\begin{tabular}{|c|c|c|c|c|c|c|}
\hline \multirow[t]{3}{*}{ Variáveis } & \multicolumn{4}{|c|}{ Grupo } & \multicolumn{2}{|c|}{ Total } \\
\hline & \multicolumn{2}{|c|}{ Crianças } & \multicolumn{2}{|c|}{ Adolescentes } & \multirow[b]{2}{*}{$\mathrm{n}$} & \multirow[b]{2}{*}{$\%$} \\
\hline & $\mathbf{n}$ & $\%$ & $\mathrm{n}$ & $\%$ & & \\
\hline \multicolumn{7}{|l|}{ Tipo de violência } \\
\hline \multicolumn{7}{|l|}{ Física } \\
\hline Feminino & 20 & 6,6 & 70 & 23,1 & 90 & 29,7 \\
\hline Masculino & 26 & 8,6 & 42 & 13,9 & 68 & 22,5 \\
\hline Subtotal & 46 & 15,2 & 112 & 37,0 & 158 & 52,2 \\
\hline \multicolumn{7}{|l|}{ Sexual } \\
\hline Feminino & 54 & 17,8 & 77 & 25,4 & 131 & 43,2 \\
\hline Masculino & 13 & 4,3 & 1 & 0,3 & 14 & 4,6 \\
\hline Subtotal & 67 & 22,1 & 78 & 25,7 & 145 & 47,8 \\
\hline Total & 113 & 37,3 & 190 & 62,7 & 303 & 100,0 \\
\hline \multicolumn{7}{|l|}{ Gravidade da violência física } \\
\hline Lesão leve & 34 & 21,5 & 95 & 60,1 & 129 & 81,6 \\
\hline Lesão grave & 8 & 5,1 & 10 & 6,3 & 18 & 11,4 \\
\hline Lesão gravíssima & 0 & 0,0 & 1 & 0,6 & 1 & 0,6 \\
\hline Sem sinais de violência & 4 & 2,5 & 6 & 3,9 & 10 & 6,4 \\
\hline Total & 46 & 29,1 & 112 & 70,9 & 158 & 100,0 \\
\hline \multicolumn{7}{|l|}{ Tipo de violência sexual } \\
\hline Estupro & 5 & 3,4 & 14 & 9,7 & 19 & 13,1 \\
\hline Estupro presumido & 1 & 0,7 & 19 & 13,1 & 20 & 13,8 \\
\hline Atentado violento & 4 & 2,8 & 0 & 0,0 & 4 & 2,8 \\
\hline Ato libidinoso & 15 & 10,3 & 7 & 4,8 & 22 & 15,1 \\
\hline Sem sinais de violência & 42 & 29,0 & 38 & 26,2 & 80 & 55,2 \\
\hline Total & 67 & 46,2 & 78 & 53,8 & 145 & 100,0 \\
\hline \multicolumn{7}{|l|}{ Local da ocorrência } \\
\hline Casa da vítima & 46 & 15,2 & 36 & 11,9 & 82 & 27,1 \\
\hline Próximo da casa & 12 & 4,9 & 40 & 13,3 & 52 & 18,2 \\
\hline Casa de familiar & 4 & 1,3 & 4 & 1,3 & 8 & 2,6 \\
\hline Casa do agressor & 21 & 6,9 & 25 & 8,3 & 46 & 15,2 \\
\hline Casa de terceiro & 7 & 2,3 & 8 & 2,6 & 15 & 4,9 \\
\hline Via pública & 9 & 3,0 & 44 & 14,5 & 53 & 17,5 \\
\hline Escola & 7 & 2,3 & 14 & 4,6 & 21 & 6,9 \\
\hline Motel & 0 & 0,0 & 5 & 1,6 & 5 & 1,6 \\
\hline Indeterminado & 7 & 2,3 & 11 & 3,6 & 18 & 5,9 \\
\hline Outros & 0 & 0,0 & 3 & 1,0 & 3 & 1,0 \\
\hline Total & 113 & 37,3 & 190 & 62,7 & 303 & 100,0 \\
\hline \multicolumn{7}{|l|}{ Identificação do agressor } \\
\hline Pai & 16 & 5,3 & 8 & 2,6 & 24 & 7,9 \\
\hline Mãe & 7 & 2,3 & 2 & 0,6 & 9 & 2,9 \\
\hline Padrasto & 21 & 6,9 & 15 & 5,0 & 36 & 11,9 \\
\hline Familiar & 13 & 4,3 & 6 & 2,0 & 19 & 6,3 \\
\hline Conhecido & 35 & 11,6 & 78 & 25,7 & 113 & 37,3 \\
\hline Desconhecido & 6 & 2,0 & 24 & 8,0 & 30 & 10,0 \\
\hline Companheiro(a) & 3 & 1,0 & 28 & 9,2 & 31 & 10,2 \\
\hline Vizinhos & 11 & 3,6 & 24 & 8,0 & 35 & 11,6 \\
\hline Não informado & 0 & 0,0 & 3 & 1,0 & 3 & 1,0 \\
\hline Outros & 1 & 0,3 & 2 & 0,6 & 3 & 1,0 \\
\hline Total & 113 & 37,3 & 190 & 62,7 & 303 & 100,0 \\
\hline
\end{tabular}




\section{Resumo}

O objetivo do estudo foi descrever as características da violência contra crianças e adolescentes atendidos no Instituto Médico-Legal de Maceió (IML/Maceió), Alagoas, Brasil. Entrevistaram-se 303 vítimas de violência, submetidas a exame de corpo de delito, de setembro/2008 a março/2009. Coletaram-se dados sobre tipo de violência, sexo, idade, escolaridade e classificação econômica da vítima; escolaridade da mãe; identificação do agressor e do denunciante e local da violência. A violência contra crianças e adolescentes que chegam ao IML/Maceió está concentrada nas camadas sociais mais baixas e tem nas meninas e jovens do sexo feminino as principais vítimas. Os agressores foram, na maioria, pessoas conhecidas da família. Predominaram casos de violência sexual entre as crianças e, física entre os adolescentes. A casa da vítima foi o local mais comum para a prática da violência. Estudos adicionais devem ser realizados para identificar se a violência contra crianças e adolescentes nos demais segmentos sociais não existe ou apenas não demanda exames de corpo de delito, o que contribui para sua invisibilidade.

Agressão; Violência; Violência Sexual; Adolescente; Criança

\section{Colaboradores}

J. A. T. L. Guimarães contribuiu com a pesquisa bibliográfica, elaboração de projeto de pesquisa, coleta e análise de dados e escrita do artigo. W. V. Villela contribuiu com a orientação metodológica e teórica, correção e revisão do artigo.

\section{Referências}

1. Minayo MCS. A difícil e lenta entrada da violência na agenda do setor saúde. Cad Saúde Pública 2004; 20:646-7.

2. De Lorenzi DRS, Pontalti L, Flech RM. Maus tratos na infância e adolescência: análise de 100 casos. Rev Cient AMECS 2001; 10:47-52.

3. Bazon MR. Violências contra crianças e adolescentes: análise de quatro anos de notificações feitas ao Conselho Tutelar na cidade de Ribeirão Preto, São Paulo, Brasil. Cad Saúde Pública 2008; 24:323-32.

4. Gomes R, Minayo MCS, Silva CFR. Violência contra a mulher: uma questão transnacional e transcultural das relações de gênero. In: Secretaria de Vigilância em Saúde, Ministério da Saúde, organizador. Impacto da violência na saúde dos brasileiros. Brasília: Ministério da Saúde; 2005. p. 117-40.
5. Cavalcanti AL, Duarte RC. Perfil da criança e do adolescente vítimas de violência doméstica. Rev Bras Ciênc Saúde 2004; 8:183-90.

6. Araújo MF. Violência e abuso sexual na família. Psicol Estud 2002; 7:3-11.

7. Vendruscolo TS, Ferriani MGC, Silva MAI. As políticas públicas de atendimento à criança e ao adolescente vítimas de violência doméstica. Rev Latinoam Enferm 2007; 15(n. spe.):812-9.

8. Noronha CV, Machado EP, Tapparelli G, Cordeiro TRF, Laranjeira DHP, Santos CAT. Violência, etnia e cor: um estudo dos diferenciais na região metropolitana de Salvador, Bahia, Brasil. Rev Panam Salud Pública 1999; 5:268-77. 
9. Rickert VI, Wiemann CM, Vaughan RD, White JW. Rates and risk factors for sexual violence among an ethnically diverse sample of adolescents. Arch Pediatr Adolesc Med 2004; 158:1132-9.

10. Gomes MLM, Falbo Neto GH, Viana CH, Silva MA. Perfil clínico-epidemiológico de crianças e adolescentes do sexo feminino vítimas de violência atendidas em um serviço de apoio à mulher, Recife, Pernambuco. Rev Bras Saúde Matern Infant 2006; 6 Suppl 1:S27-34.

11. Pires JM, Goldani MZ, Vieira EM, Nava TR, Feldens L, Castilhos K, et al. Barreiras, para notificação pelo pediatra, de maus-tratos infantis. Rev Bras Saúde Matern Infant 2005; 5:103-8.

12. Reichenheim ME, Hasselmann MH, Moraes CL. Conseqüências da violência familiar na saúde da criança e do adolescente: contribuições para a elaboração de propostas de ação. Ciênc Saúde Coletiva $1999 ; 4: 109-21$.

13. Morales AE, Schramm FR. A moralidade do abuso sexual intra-familiar em menores. Ciênc Saúde Coletiva 2002; 7:265-73.
14. Shrier LA, Pierce JD, Emans SJ, Durant RH. Gender differences in risk behaviors associated with forced or pressured sex. Arch Pediatr Adolesc Med 1998; 152:57-63.

15. Monteiro CFS, Teles DCBS, Castro KL, Vasconcelos NSV, Magalhães RLB, Deus MCBR. Violência sexual contra criança no meio intra-familiar atendidos no SAMVVIS, Teresina, PI. Rev Bras Enferm 2008; 61:459-63.

16. Costa MCO, Carvalho RC, Santa Bárbara JFR, Santos CAST, Gomes WA, Souza HL. O perfil da violência contra crianças e adolescentes, segundo registros de Conselhos Tutelares: vítimas, agressores e manifestações de violência. Ciênc Saúde Coletiva 2007; 12:1129-41.

Recebido em 07/Mar/2010

Versão final reapresentada em 24/Mai/2011

Aprovado em 22/Jun/2011 